\title{
Efficient ADCC killing of meningioma by avelumab and a high-affinity natural killer cell line, haNK
}

\author{
Amber J. Giles, ${ }^{1}$ Shuyu Hao, ${ }^{1,2}$ Michelle Padget, ${ }^{3}$ Hua Song, ${ }^{1}$ Wei Zhang, ${ }^{1}$ John Lynes, ${ }^{4}$ \\ Victoria Sanchez, ${ }^{4}$ Yang Liu, ${ }^{1}$ Jinkyu Jung, ${ }^{1}$ Xiaoyu Cao, ${ }^{1}$ Rika Fujii, ${ }^{3}$ Randy Jensen, ${ }^{5}$ David Gillespie, \\ Jeffrey Schlom, ${ }^{3}$ Mark R. Gilbert, ${ }^{1}$ Edjah K. Nduom, ${ }^{4}$ Chunzhang Yang, ${ }^{1}$ John H. Lee, ${ }^{6}$ \\ Patrick Soon-Shiong, ${ }^{6}$ James W. Hodge, ${ }^{3}$ and Deric M. Park ${ }^{1,7}$ \\ ${ }^{1}$ Neuro-Oncology Branch, Center for Cancer Research, National Cancer Institute, National Institutes of Health, Bethesda, \\ Maryland, USA. ${ }^{2}$ Neurosurgical Department, Beijing Tiantan Hospital, Capital Medical University, Beijing, China. \\ ${ }^{3}$ Laboratory of Tumor Immunology and Biology, Center for Cancer Research, National Cancer Institute, National Institutes \\ of Health, Bethesda, Maryland, USA. ${ }^{4}$ Surgical Neurology Branch, National Institute of Neurological Disorders and Stroke, \\ National Institutes of Health (NIH), Bethesda, Maryland, USA. ${ }^{5}$ Department of Neurosurgery, University of Utah, Salt \\ Lake City, Utah, USA. ${ }^{6}$ NantKwest, Culver City, California, USA. 'Department of Neurology and the Committee on Clinical \\ Pharmacology and Pharmacogenomics, The University of Chicago, Chicago, Illinois, USA.
}

\begin{abstract}
Meningiomas are the most common adult primary tumor of the central nervous system, but there are no known effective medical therapies for recurrent meningioma, particularly for World Health Organization grade II and III tumors. Meningiomas arise from the meninges, located outside the blood-brain barrier, and therefore may be directly targeted by antibody-mediated immunotherapy. We found that programmed cell death ligand 1 (PD-L1) was highly expressed in multiple human malignant meningioma cell lines and patient tumor samples. PD-L1 was targeted with the antiPD-L1 antibody avelumab and directed natural killer cells to mediate antibody-dependent cellular cytotoxicity (ADCC) of PD-L1-expressing meningioma tumors both in vitro and in vivo. ADCC of meningioma cells was significantly increased in target cells that upregulated PD-L1 expression and, conversely, abrogated in tumor cells that were depleted of PD-L1. Additionally, the highaffinity natural killer cell line, haNK, outperformed healthy donor NK cells in meningioma ADCC. Together, these data support a clinical trial designed to target PD-L1 with avelumab and haNK cells, potentially offering a novel immunotherapeutic approach for patients with malignant meningioma.
\end{abstract}

Authorship note: AJC and SH contributed equally to this work.

Conflict of interest: JHL is an employee of NantKwest. PSS is founder of and is an executive of NantKwest.

Copyright: () 2019, American Society for Clinical Investigation.

Submitted: May 29, 2019 Accepted: September 11, 2019 Published: October 17, 2019.

Reference information: /CI Insight. 2019;4(20):e130688. https://doi.org/10.1172/jci. insight.130688.

\section{Introduction}

Meningiomas are the most common primary tumor of the CNS (36.1\%), occurring in 7.61 per 100,000 individuals (1). Most cases are low grade (World Health Organization [WHO] grade I), and durable responses or cures can be achieved with surgery alone. However, atypical (WHO grade II) and anaplastic (WHO grade III) meningiomas frequently recur following resection, and recurrent tumors are often more aggressive $(2,3)$. The 5 -year overall survival rate for patients with anaplastic meningioma is $35 \%-61 \%$ (4-6), and no effective treatment options are available for patients with these aggressive tumors.

Immunotherapy has become an important option for patients with solid tumors, such as melanoma (7). However, antitumor immunity is hindered by programmed cell death ligand 1 (PD-L1) on immune-suppressing cells and tumors. Antibody-mediated PD-L1 blockade has shown clinical benefit in locally advanced or metastatic bladder cancer, metastatic non-small cell lung cancer (NSCLC), melanoma, renal cell cancer, and ovarian cancer (8-11). Avelumab, a fully human anti-PD-L1 IgG $_{1}$ antibody, has shown clinical efficacy against solid tumors and $\operatorname{NSCLC}(12,13)$. In addition to blocking the immune-suppressing function of PD-L1, avelumab can mediate antibody-dependent cellular cytotoxicity (ADCC) of tumor cells by cytotoxic T cells and natural killer (NK) cells $(14,15)$.

Tumor killing by ADCC is induced when CD16 on cytotoxic effector cells, such as NK cells, ligates the Fc portion of target-bound antibody. NK cells are potent killers that contain large amounts of perforin and granzymes within cytotoxic granules. In addition to direct tumor cell lysis, NK cells can release 
cytokines, including interferon- $\gamma$ (IFN- $\gamma$ ), that further enhance the adaptive immune response. Yet, NK function and activity vary greatly among individuals due, in part, to genetic polymorphisms of Fc receptors that modulate NK activation. Patient NK cells that express the high-affinity CD16 valine (V) allele (V/V) experience better clinical outcomes than those with the lower affinity $\mathrm{V} /$ phenylalanine $(\mathrm{F})$ and $\mathrm{F} / \mathrm{F}$ genotypes. Reports vary as to what percentage of the population is of the V/V genotype, but most studies report approximately $10 \%-20 \%(16-20)$.

The activated NK line (aNK) NK-92 (21-23) has been shown to mediate ADCC in vivo. This parental line was engineered to express the high-affinity (ha) $\mathrm{Fc}_{\mathrm{C}}$ receptor $(\mathrm{V} / \mathrm{V})$, enhancing cytotoxic activity and generating the haNK cell line. In addition, haNK cells were engineered to express IL-2 and granzyme B. As with healthy donor NK cells, haNK cells do not require recipient matching, making these cells an attractive and continuous source of off-the-shelf cytotoxic mediators in immunotherapy.

PD-L1 has been detected on meningioma cells from surgical resections $(24,25)$. Here, we demonstrate that patient-derived malignant cell lines constitutively express high levels of PD-L1 under basal conditions compared with low-grade meningioma and other cancer cell lines. The PD-L1-specific antibody, avelumab, directed meningioma cell lysis by healthy donor NK cells. Tumor killing was mediated by ADCC because it was prevented by CD16 blockade and enhanced with healthy donor NK cells expressing the high-affinity CD16 receptor (haNK). The engineered aNK cell line, haNK, lysed meningioma cells more effectively than healthy donor NK cells when cultured with avelumab. Tumor cell killing was further enhanced with biologically relevant conditions of IFN- $\gamma$ exposure or hypoxia that upregulated PD-L1 surface expression and was abrogated when PD-L1 was deleted from tumor lines. These findings translated into a survival benefit in vivo using an orthotopic skull base model or subcutaneously implanted meningioma cells and treating with avelumab and haNK. Our studies suggest that an immunotherapy using avelumab-directed ADCC against malignant meningioma may offer a novel treatment approach for this cancer with no currently available effective treatment.

\section{Results}

Basal PD-L1 expression was quantified by flow cytometry on 2 prostate cancer, 2 breast cancer, 2 lung carcinoma, 4 chordoma, 3 low-grade meningioma, and 5 malignant meningioma cell lines (Figure 1, A and $\mathrm{B})$. PD-L1 expression was highest on malignant meningioma cell lines and 2-fold greater than that of the next highest cell line, HCC4006. Meningioma expression was next confirmed in tumor blocks from patients with meningioma. Diffuse PD-L1-positive cells were detected in lower grade samples whereas clusters of PD-L1-positive cells and increased overall positive staining on tumor cells were detected in higher grade specimens (Figure 1C). PD-L1 was previously used to target chordoma tumor cell lines for ADCC by using NK cells and the anti-PD-L1 antibody avelumab (14). Stronger PD-L1 expression on chordoma tumor cells correlated with greater susceptibility to avelumab-directed ADCC of other tumor cells by peripheral blood mononuclear cells (PBMCs) and NK cells (15). Because the average PD-L1 intensity of meningioma was over 10-fold greater than in chordoma, its potential to target meningioma for ADCC with avelumab was further investigated.

The PD-1/PD-L1 axis has been reported to be potentially suppressive to NK cell function (26-29). We examined healthy donor unactivated NK cells or haNK cells for the surface expression of PD-L1 (CD274), PD1 (CD279), and additional suppressive markers NKG2/KLRD1 (CD94)and KIR2DL1/2/3 (CD158), as well as B7-1 (CD80) (Supplemental Table 1; supplemental material available online with this article; https:// doi.org/10.1172/jci.insight.130688DS1). For 4 healthy donors, the NK cell expression of PD-L1 ranged from $3 \%$ to $21 \%$. haNK cells had a surface expression of $60 \%$. We hypothesized that ADCC mediated by the anti-PD-L1 IgG 1 avelumab would ameliorate potential suppression (30). Avelumab or healthy donor NK cells as single agents induced minimal meningioma cell lysis, as measured by indium release from tumor cells. However, the combination of avelumab with healthy donor NK cells lysed IOMM, GAR, and CH-157 cell lines with increasing effector-to-target ratios (Figure 2A). Importantly, these NK cells were not preactivated with cytokines. The degree of lysis was dependent on the donor used and potentially affected by the expression of inhibitory ligands on a tumor cell. We interrogated IOMM cells for surface expression of molecules potentially inhibitory to NK cells: programmed cell death 1 (PD-1; CD279), PD-L1 (CD274), HLAABC, MIC A/B, and CEACAM1 (CD66 a/c/e) (Supplemental Table 1B). These cells expressed no PD-1, MIC A/B, or CEACAM1; however, they expressed substantial levels of PD-L1 and HLA-ABC. Despite this, NK cells were able to effectively lyse this tumor in the presence of avelumab (Figure 1A). 
A
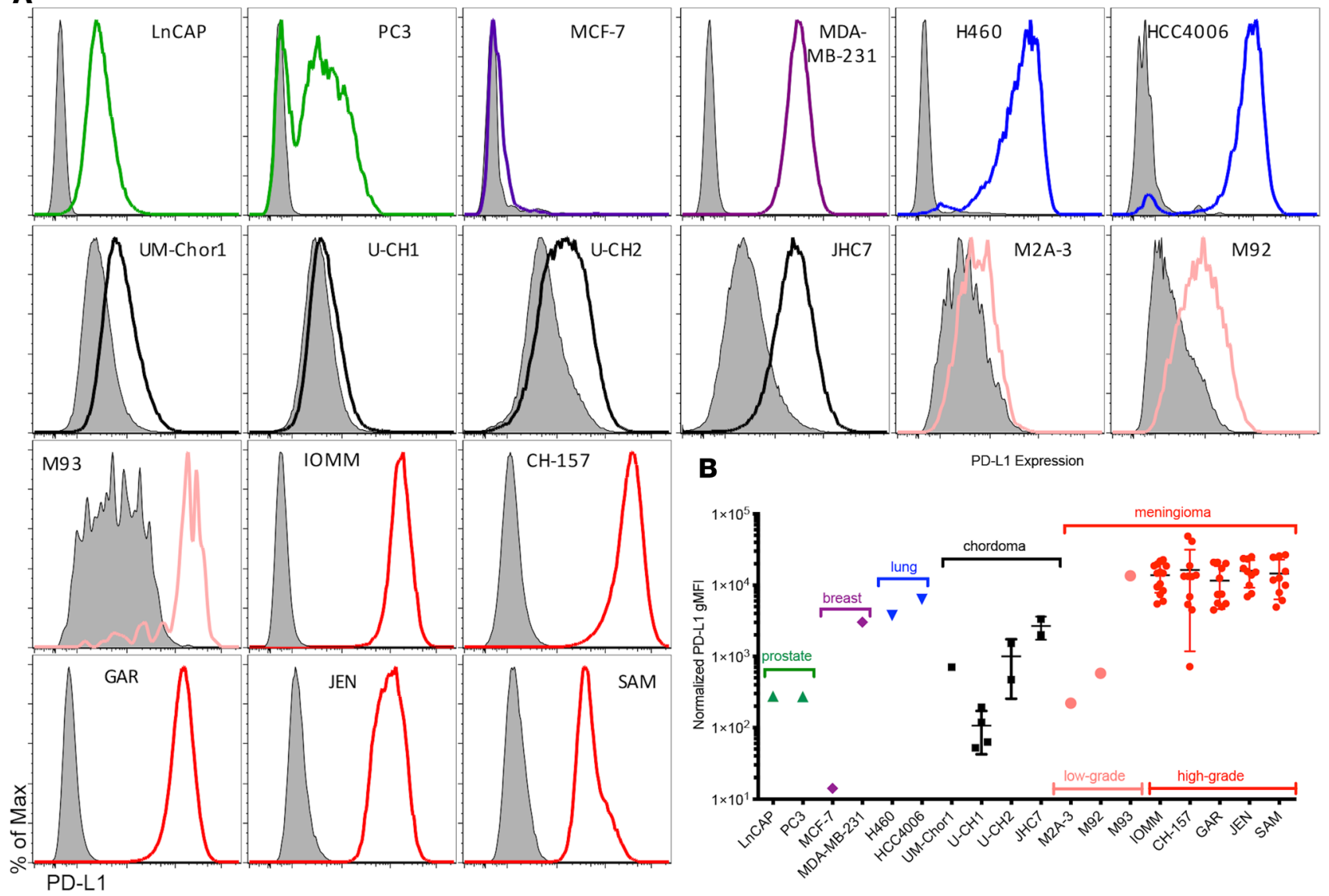

B

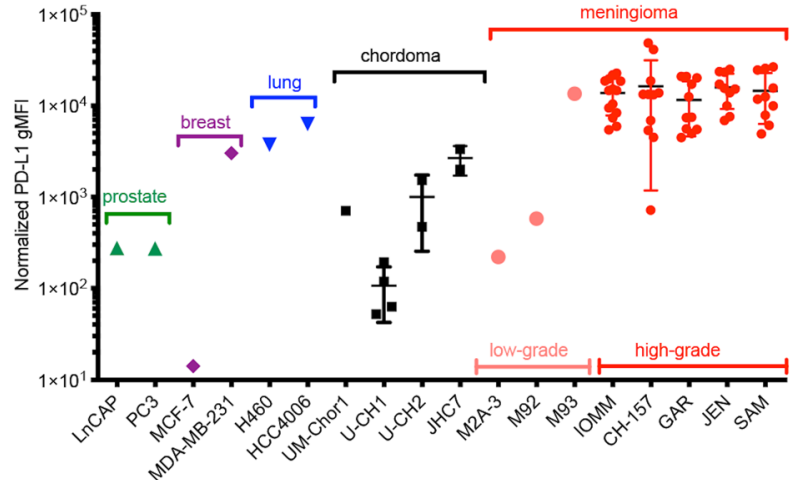

C Grade I Grade II Grade III
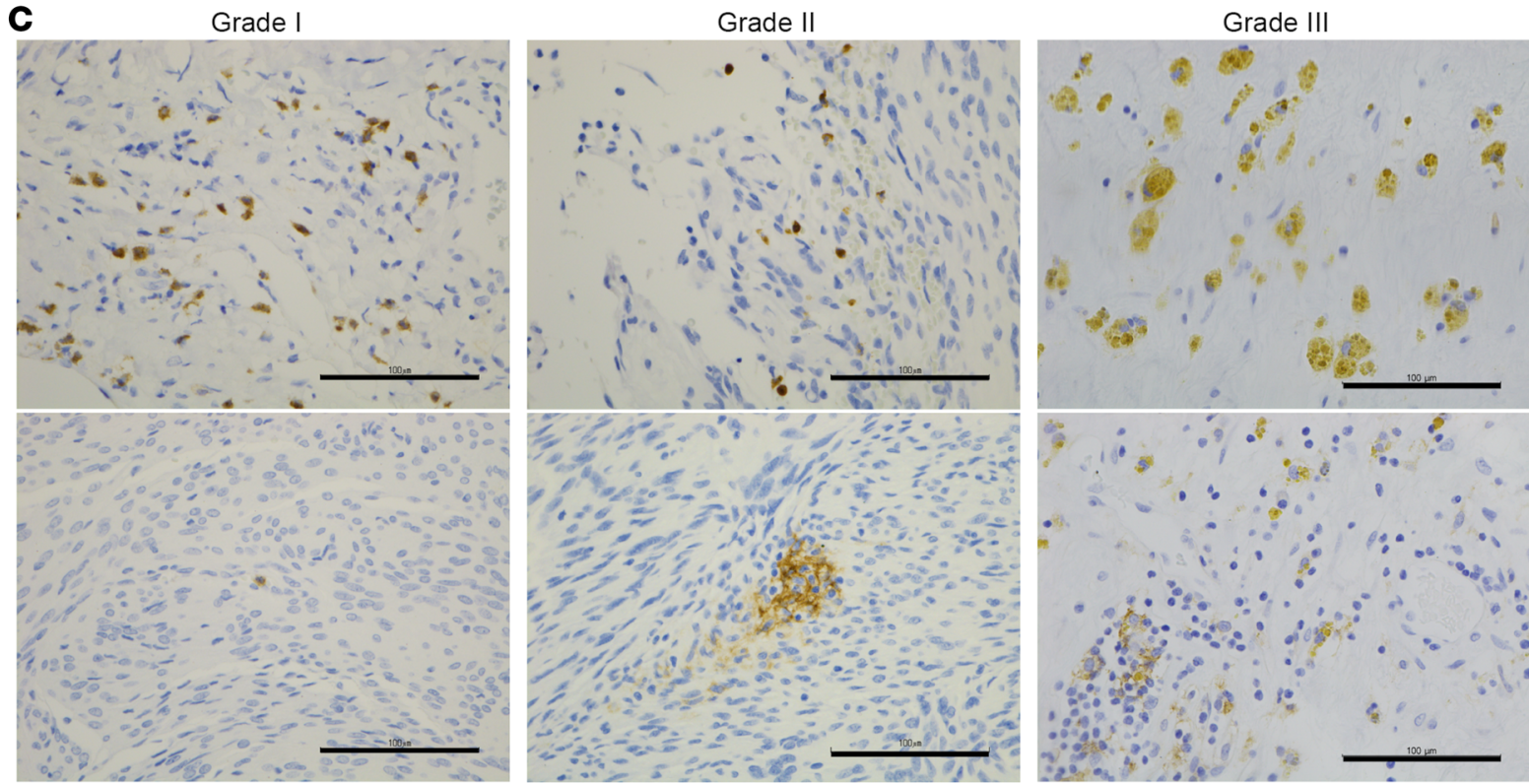

Figure 1. PD-L1 is highly expressed on malignant meningioma compared with other tumor cell lines. (A) Representative flow cytometry plots of the indicated cell lines. PD-L1 expression is shown on single-gated, viable cells. The fluorescence minus 1 (FMO) control stain without PD-L1 antibody is shown for each cell line in gray. (B) PD-L1 intensity was normalized by subtracting the geometric mean fluorescence intensity (gMFI) of PD-L1 for the FMO sample from the PD-L1 gMFI of the matched stained sample. Each symbol represents an individual sample; multiple symbols represent separate runs. (C) PD-L1 immunohistochemistry of a meningioma tumor block from a patient with grade I (first column), grade II (middle column), or grade III (last column) tumors. Two areas are shown for each tumor under original magnification $\times 40$. Scale bar: $100 \mu \mathrm{m}$. 
A

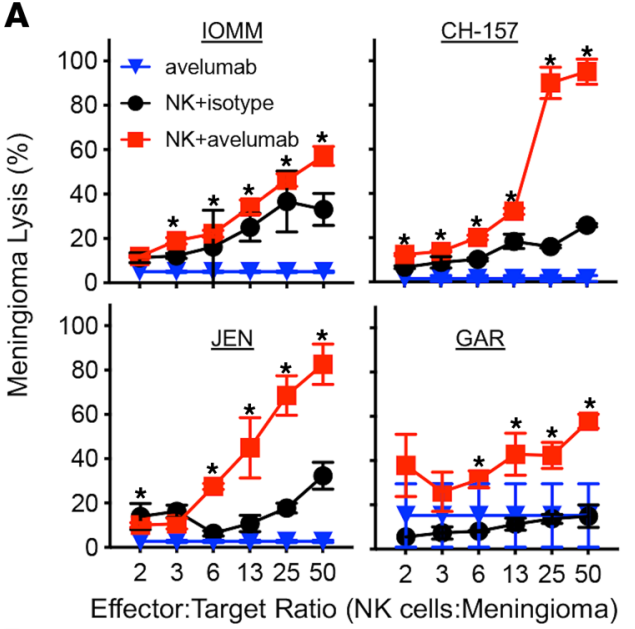

B

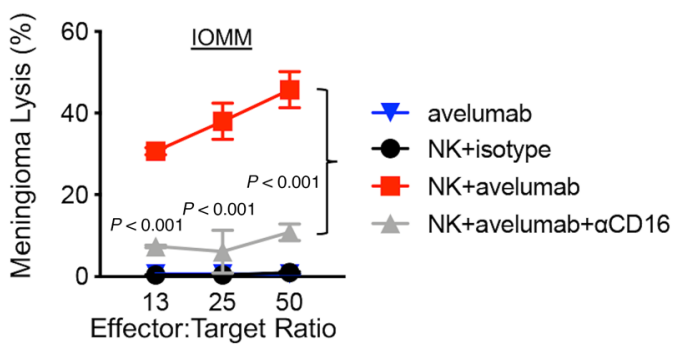

Figure 2. ADCC of meningioma is mediated by Fc receptor binding, and ADCC is increased with high-avidity Fc receptors. (A) ADCC assay of malignant meningioma lines by healthy donor NK cells. Meningioma lines were incubated with avelumab alone, healthy donor NK cells and isotype antibody, or NK cells and avelumab. The ratio of NK to meningioma cells is indicated. Each sample was plated in triplicate. Data were analyzed with a 2 -way ANOVA; ${ }^{*} P<0.05$. Shown are representative data from 3 repeats using different healthy donors. (B) ADCC of IOMM incubated with avelumab or healthy donor NK cells with isotype, avelumab, or avelumab with $\alpha$-CD16 (Fc-blocking) antibody. Data were analyzed with a 2-way ANOVA. (C) ADCC assay of 5 meningioma lines by NK cells from healthy donors with the F/F or V/V polymorphism of the CD16 receptor (effector/ target $[E / T]$ of 10:1). Data were analyzed with unpaired 1-tailed $t$ test comparing NK and isotype with NK and avelumab conditions. (D) Lysis of IOMM meningioma by healthy donor NK cells versus haNK cells in the presence of avelumab. Samples were plated in triplicate. Data were analyzed with 1-way ANOVA.

C
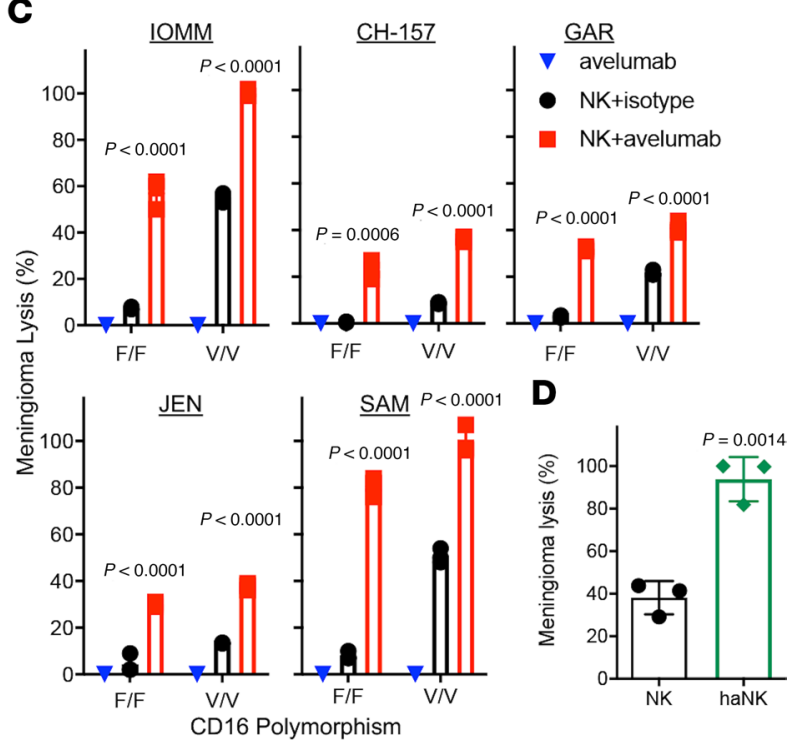

To confirm that meningioma cells were lysed by NK cells via ADCC, a CD16-blocking (FcyRIIIa-blocking) antibody was used to prevent $\mathrm{CD} 16$ on NK cells from binding the Fc portion of avelumab. Blocking CD16 resulted in an $80 \%$ decrease in meningioma lysis, indicating that cell lysis was due to ADCC (Figure 2B).

A single nucleotide polymorphism of CD16 at position 158 affects the affinity of $\mathrm{CD} 16$ for the $\mathrm{Fc}$ region of an antibody. NK cells from individuals with a valine (V) at position 158 (Fc $\gamma$ RIIIa-158V) bind the Fc region of $\mathrm{IgG}_{1}$ with greater affinity than those with phenylalanine (F) at this position (FcyRIIIa-158F) (31). A stronger binding affinity of the $\mathrm{Fc}_{\mathrm{c}}$ receptor on cytotoxic cells translates into a higher magnitude of $\mathrm{ADCC}$ response toward antibody-coated target cells. This has clinical implications because FcyRIIIa-158V/V expression correlates with longer progression-free survival for patients receiving cetuximab or trastuzumab $(16,19)$. In all meningioma lines tested, NK cells from V/V donors mediated greater ADCC than NK cells from $\mathrm{F} / \mathrm{F}$ donors with fold increases of 1.74 (IOMM), 1.58 (CH-157), 1.28 (GAR), 1.26 (JEN), and 1.24 (SAM) for V/V relative to F/F (Figure 2C). 
A
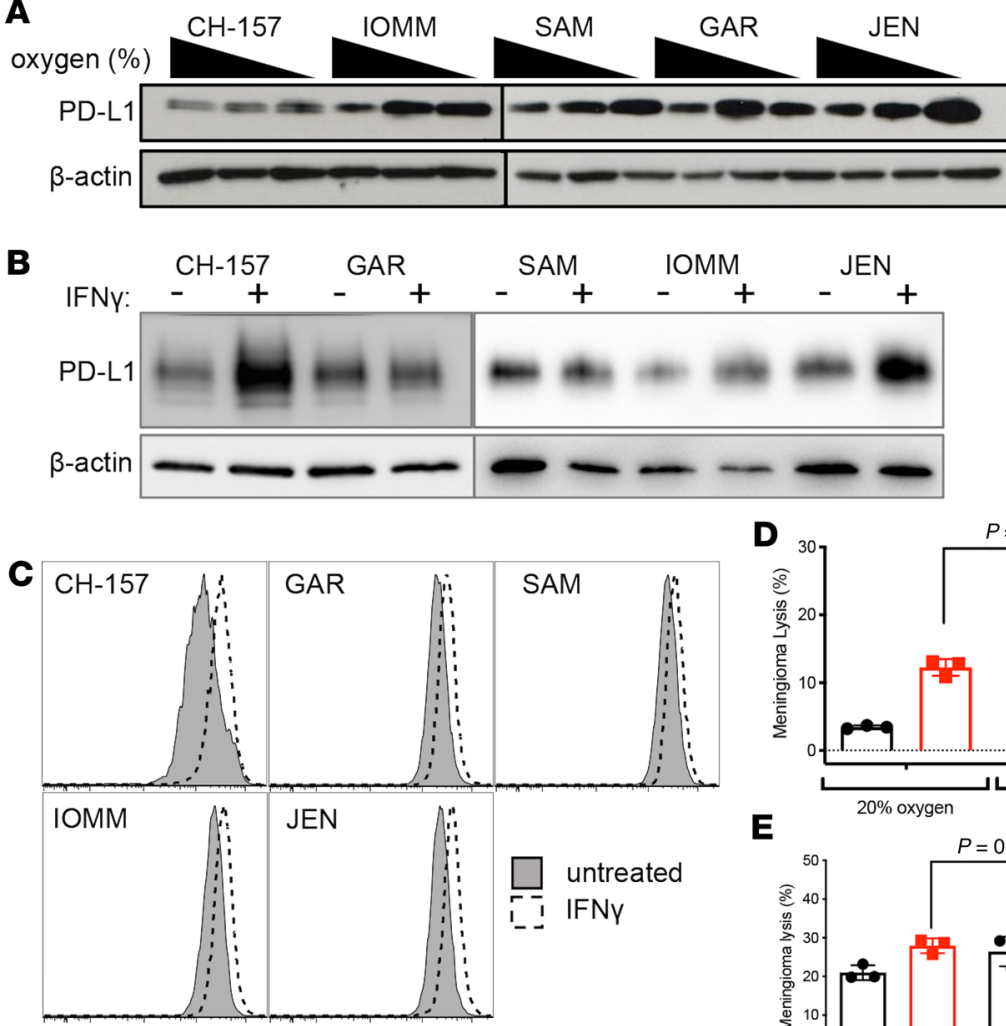

PD-L1 expression

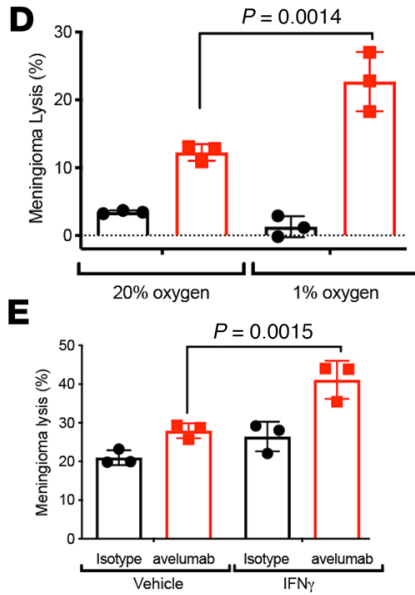

Figure 3. PD-L1, upregulated by IFN- $\gamma$ and hypoxia, enhances meningioma lysis by haNK cells. (A) Total PD-L1 protein was detected by Western blotting in 5 meningioma cell lines cultured under $20 \%, 5 \%$, or $1 \%$ oxygen. (B) Western analysis of PD-L1 protein from the indicated meningioma lines cultured with or without IFN- $\gamma$ treatment. (C) Surface PD-L1 was measured by flow cytometry for the indicated cell lines. Cells were untreated (filled gray histogram) or treated with IFN- $\gamma$ (dashed line). (D) ADCC assay of IOMM cells incubated with isotype or avelumab antibody and haNK cells under $20 \%$ or $1 \%$ oxygen (E/T of 25:1). (E) ADCC assay of IOMM cells cultured with vehicle or IFN- $\gamma$ for 2 days before incubating with isotype or avelumab antibody and haNK cells (E/T of 25:1). Each condition was plated in triplicate, and data were analyzed with a 2-way ANOVA.

Healthy donor NK cells were next compared to the haNK cell line for cytotoxic activity against IOMM meningioma cells. The haNK cell line was engineered from the non-Hodgkin lymphoma NK-92 cell line to express the high-affinity CD16 receptor and secrete high levels of IL-2. These modifications conferred advantages of IL-2-mediated haNK survival and enhanced ADCC function via the high-avidity CD16 receptor. The haNK cell line lysed malignant meningioma cells when incubated with avelumab in vitro and was more effective at ADCC compared with healthy donor NK cells (Figure 2D). Therefore, haNK cells were preferentially selected for further investigation.

Increased surface expression of PD-L1 was previously shown to enhance the efficacy of avelumab-directed ADCC against chordoma, a cancer found in the skull base and spine (14). PD-L1 is upregulated by hypoxia and IFN- $\gamma(32,33)$, which are associated with advanced grade in meningioma (34). Meningioma cell lines cultured under hypoxia upregulated total PD-L1 protein compared with basal levels (Figure 3A). Similarly, surface PD-L1 and total PD-L1 protein were increased when cells were cultured in the presence of IFN- $\gamma$ (Figure 3, B and C). As with chordoma, ADCC was increased when meningioma cells were cultured under biologically relevant conditions that upregulated PD-L1 (Figure 3, D and E). These data indicate that avelumab-mediated ADCC by haNK cells may be increased in vivo in meningioma tumors that upregulate PD-L1 during hypoxia or IFN- $\gamma$ exposure.

The ability of haNK and avelumab to mediate ADCC was next investigated in vivo using IOMM meningioma cells that were stably transduced with GFP-luciferase for longitudinal imaging, detection of viable tumor, and assessment of tumor burden (Figure 4A). Athymic mice implanted with IOMM meningioma in an orthotopic skull base model had a median survival of 21 days with vehicle treatment, similar to 
A

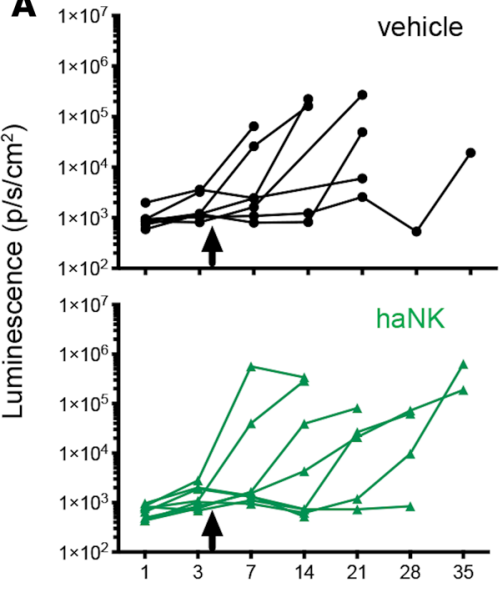

C

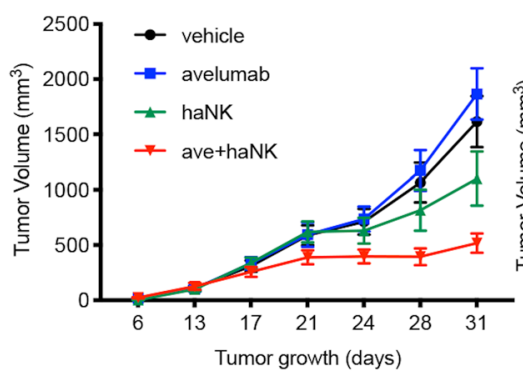

vehicle
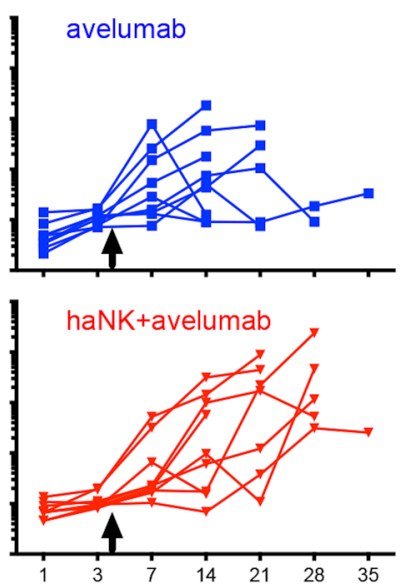

D

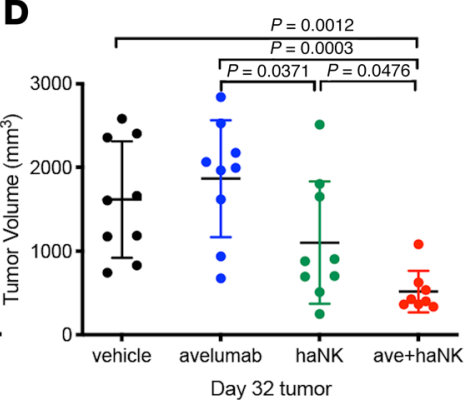

E
B
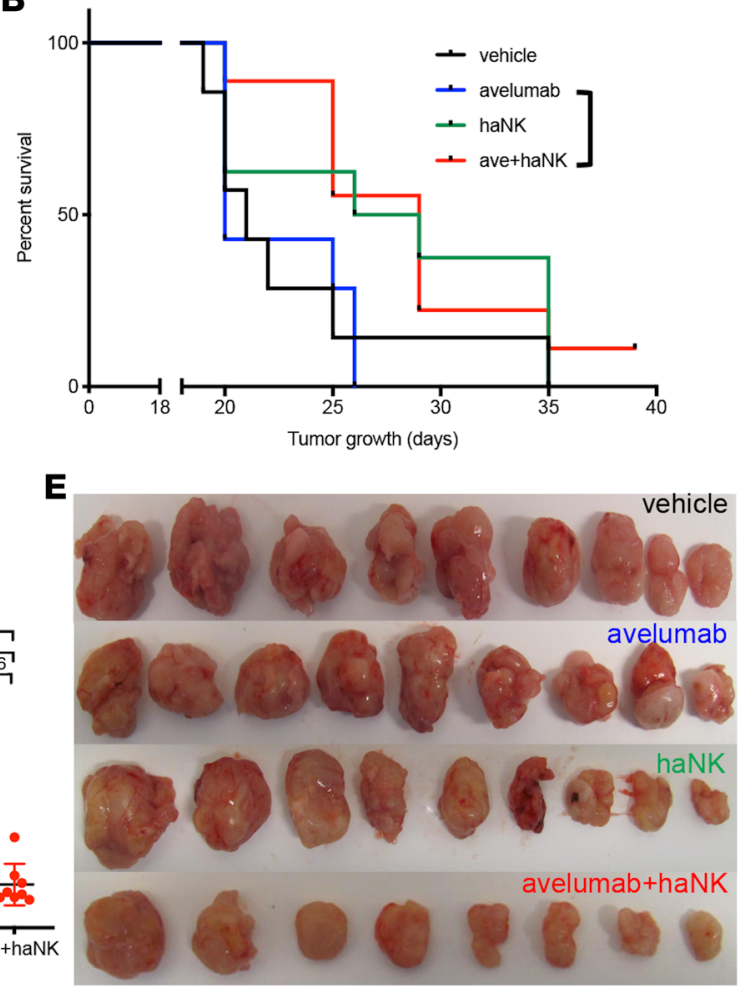

avelumab + haNK
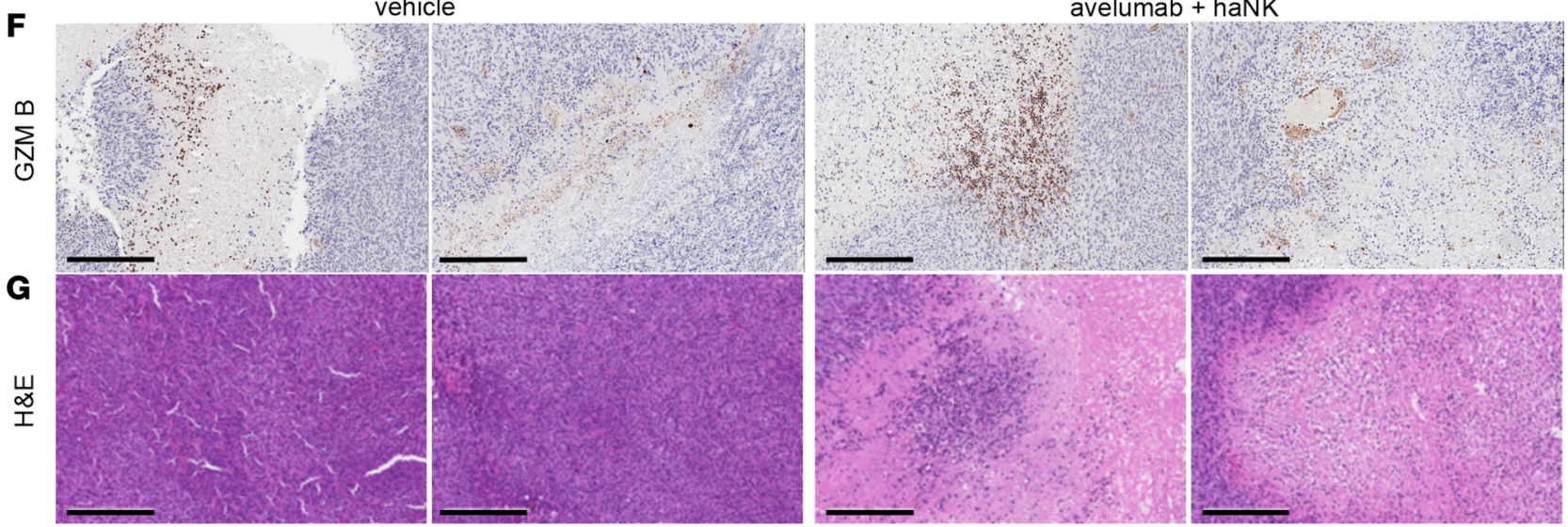

Figure 4. Avelumab and haNK treatment extends survival in meningioma tumor-bearing mice. (A and B) Female athymic nude mice were implanted with IOMM meningioma cells in a skull base model. Mice were randomized to the indicated cohorts with 9-10 mice per group. Treatment commenced 4 days following tumor implantation. Mice received 7 treatments. (A) Luminescence from GFP-luciferase-expressing IOMM tumor cells was quantified weekly. Each line represents an individual mouse. Arrows indicate commencement of treatment. P/s/cm², photons $/ \mathrm{s} / \mathrm{cm}^{2}$. (B) Kaplan-Meier survival analysis of tumor-bearing mice. (C-E) IOMM meningioma cells were implanted subcutaneously. Mice were randomized based on tumor volume and treated as in $\mathbf{A}$ and $\mathbf{B}$. Treatment commenced 17 days following tumor implantation, and mice received 6 treatments. Each cohort contained 9-10 mice. (C) Mean tumor volume with SEM is shown for each cohort. (D) Tumor volume on day 32 following tumor implantation. Each symbol represents an individual mouse, and data are displayed with mean and SD. Data were analyzed using an unpaired 1-tailed $t$ test with Welch's correction. (E) Subcutaneous tumors on day 32 of tumor growth. (F) Granzyme B (CZMB) was detected by immunohistochemistry in subcutaneous meningioma tumors on day 24 of tumor growth. Vehicle-treated (left, $n=2$ ) or combination-treated (right, $n=2$ ) are shown at original magnification $\times 20$. Scale bar: $200 \mu \mathrm{m}$. (G) Hematoxylin and eosin (H\&E) stain of serial sections from tumor samples in F. Scale bar: $200 \mu \mathrm{m}$.

avelumab treatment alone (median survival of 20 days) (Figure 4B). Mice treated with haNK cells demonstrated a survival benefit (median survival of 27.5 days), and this was further enhanced in mice treated with avelumab and haNK cells (median survival of 29 days). The combination of avelumab and haNK also extended survival of mice bearing orthotopic, intracranial malignant meningioma tumors compared with avelumab alone. However, the rapid time to euthanasia in this intracranial model restricted the available window to evaluate treatment efficacy. Therefore, a subcutaneous IOMM meningioma model was used. 
A

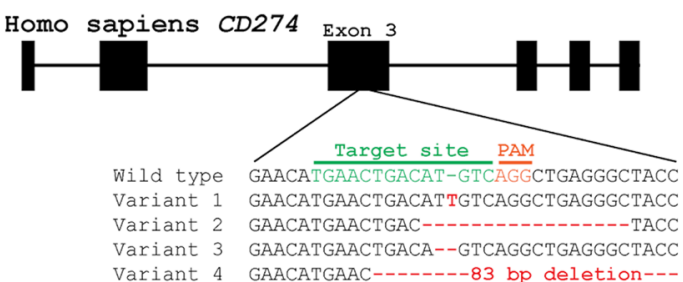

B

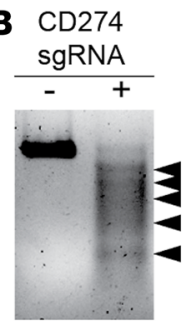

C

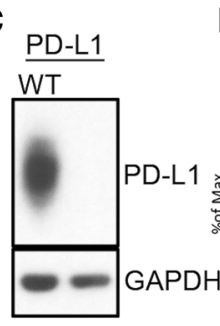

D

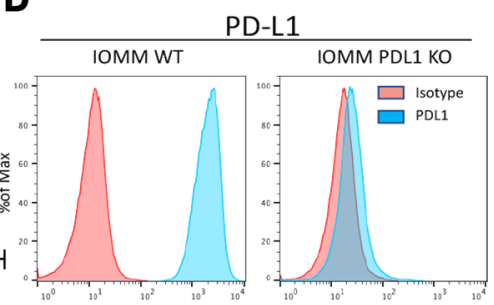

E

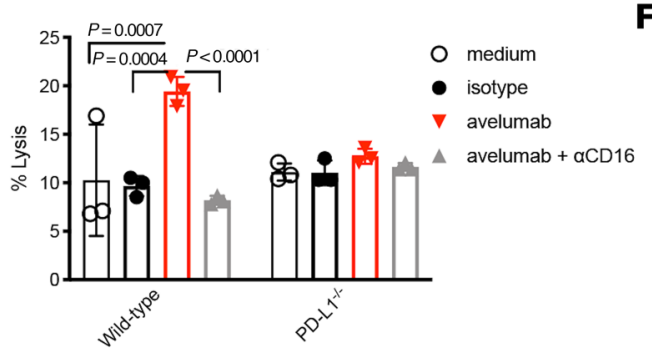

$\mathbf{F}$

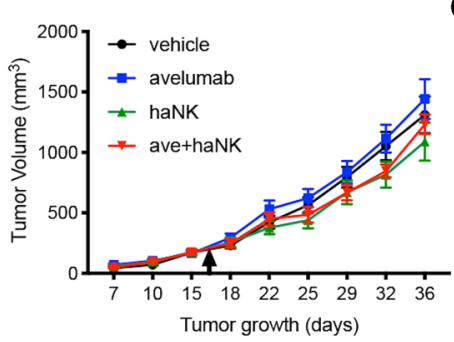

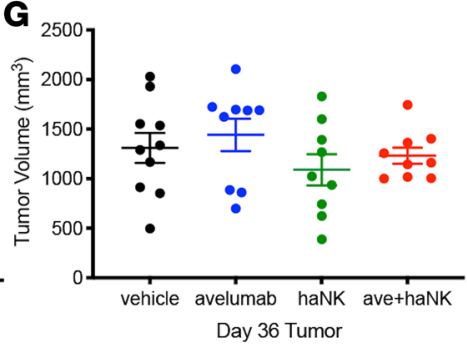

H

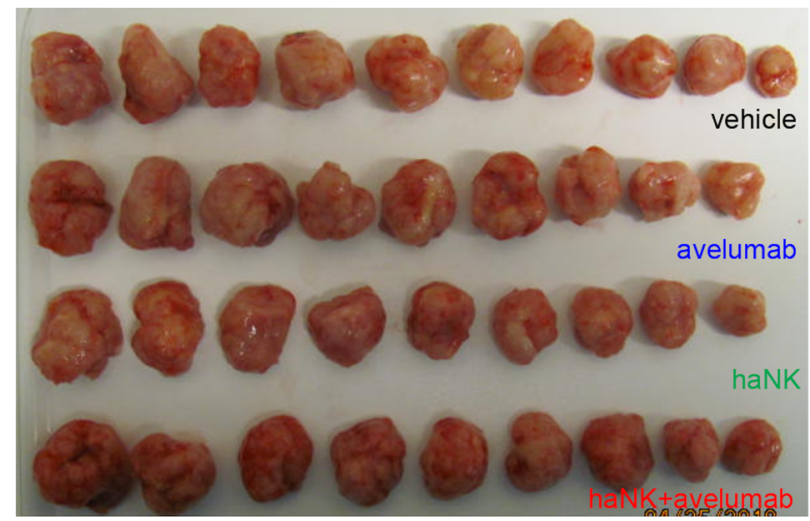

I

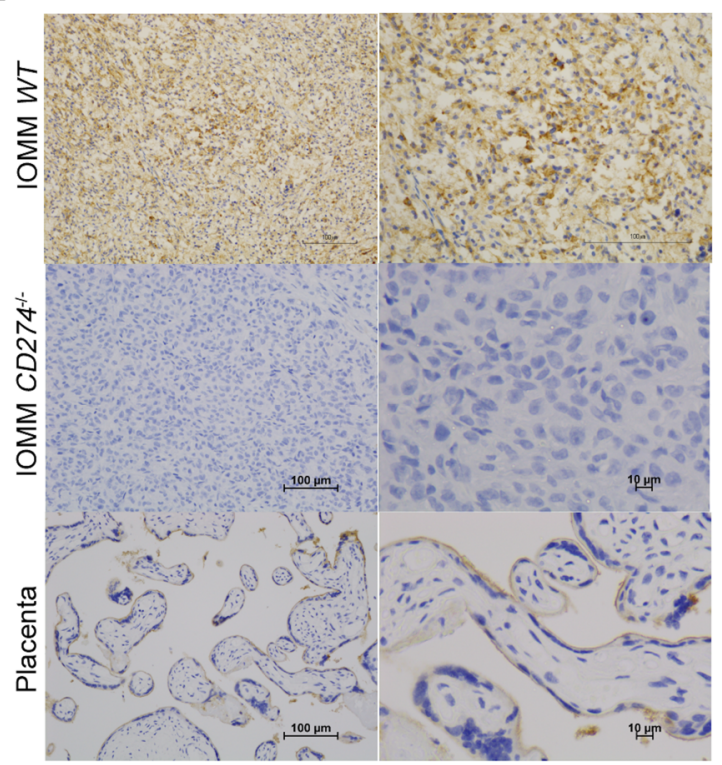

Figure 5. PD-L1 is necessary for avelumab-directed, haNK-mediated ADCC of meningioma. (A) Schematic illustration for CRISPR/Cas9-knockout strategy. Single-guide RNA (sgRNA, shown in green) was designed to target the genetic sequence of CD274 exon 3. The protospacer adjacent motif (PAM) is shown in orange. Genotypes of resultant clone variants 1-4 are shown. (B) Surveyor assay shows successful cleavage of CD274 exon 3. Arrows indicate DNA fragments resulting from CRISPR targeting. (C) Western blot of parental IOMM and CD274-/- IOMM meningioma cell lysates. GAPDH was used as a loading control. (D) Flow cytometry histogram of parental (WT) and CD274 ${ }^{-/-}$(IOMM PD-L1-KO) IOMM cells stained with PD-L1 (blue histograms) or isotype control (red histograms). (E) ADCC assay of parental or CD274-/- IOMM cells exposed to the indicated conditions. Each condition was run in triplicate. Data were analyzed with an unpaired 1-tailed $t$ test. (F-H) $C D 274^{-/-1 O M M}$ meningioma cells were implanted subcutaneously. Female athymic nude mice were randomized based on tumor volume into the indicated cohorts with $n=10$ mice/group. Treatment commenced on day 16 following tumor implantation. Mice received 8 treatments. (F) Mean tumor volume with SEM is shown for each cohort. (G) Tumor volume on day 36 following tumor implantation. Each symbol represents an individual mouse, and data are displayed with mean and SD. Data were analyzed using a 1-way ANOVA with Welch's correction. (H) Subcutaneous tumors on day 36 of tumor growth. (I) PD-L1 IHC of an IOMM-CD274-/- subcutaneous xenograft tumor (top) and normal human placenta (bottom; positive control). Images were taken with original magnification $\times 20$ (left) or $\times 40$ (right) magnification.

As in patients and in the orthotopic skull base model, subcutaneously implanted IOMM tumors lack a blood-brain barrier (35), and therefore avelumab antibody may enter the tumor microenvironment. However, the subcutaneous model circumvents the short survival window of the skull base murine model because of limited intracranial space and permits quantification of tumor volume.

Mice implanted with subcutaneous IOMM tumors and treated with the combination of avelum$\mathrm{ab}$ and haNK cells had significantly smaller tumors than any other treatment cohort (Figure 4, C-E). 
Cytotoxic immune cells were detectable in vehicle and combination avelumab and haNK-treated groups, as shown by granzyme B (GZMB) expression, likely because of haNK cells or infiltrating endogenous NK cells from athymic nude mice (Figure $4 \mathrm{~F}$ ). However, tumors from mice in the combination avelumab and haNK cohort contained larger necrotic regions compared with tumors from mice in the vehicle cohort (Figure 4G). Taken together, the combination of avelumab and haNK extended survival in both an orthotopic skull base and a subcutaneous meningioma model in conjunction with increased cytotoxic immune infiltration and tumor necrosis. These data indicate that avelumab and haNK target meningioma for ADCC in vivo.

PD-L1 was next selectively deleted from IOMM meningioma cells to address target specificity. Exon 3 of CD274, which encodes PD-L1 protein, was targeted using the CRISPR/Cas9 system. Four variants of CD274 deletion were identified (Figure 5A). PD-L1-negative cells were sorted by flow cytometry, and the efficiency of the sgRNA was examined using a Surveyor mutation detection assay (Figure 5B). Loss of PD-L1 protein was confirmed by Western blotting and flow cytometry (Figure 5, C and D, Supplemental Table 1B). Specificity of avelumab-directed ADCC was first demonstrated in vitro as IOMM-CD274 ${ }^{-1}$ cells were not lysed above background when incubated with avelumab and haNK cells (Figure 5E). Further, in vivo subcutaneous tumor growth and final tumor volume were not significantly affected by haNK or the combination avelumab and haNK in mice bearing IOMM-CD274 ${ }^{--}$tumors (Figure 5, F-H). PD-L1 deletion was maintained in IOMM$C D 274^{--}$tumors grown in vivo (Figure 5I). These data indicate that PD-L1 expression is specifically targeted by avelumab and is necessary to mediate ADCC of meningioma by haNK cells.

\section{Discussion}

PD-L1 is expressed by multiple types of tumors, enabling immune evasion (36-39). Similar to other tumors, PD-L1 expression in meningioma increases with grade and inversely correlates with immune infiltration (24). Antibodies that block PD-L1 have shown clinical benefit by preventing PD-L1 from binding the inhibitory receptor PD-1 on immune cells $(11,40,41)$. Most PD-L1-blocking antibodies are unable to mediate ADCC because of design (durvalumab and atezolizumab are Fc-deficient $\mathrm{IgG}_{1}$ ) or antibody isotype (BMS936559 is $\left.\operatorname{IgG}_{4}\right)(11,41,42)$. Yet, PD-L1 makes an attractive target for cytotoxic therapies because of high expression on tumor cells. Avelumab is a fully human $\operatorname{IgG}_{1}$ antibody that can both mediate ADCC and block the immunosuppressive PD-1/PD-L1 interaction. Although immune cells may also express PD-L1, avelumab does not deplete PD-L1-expressing immune subsets (43). Further, avelumab has shown promising efficacy in patients with various solid tumors and those with $\operatorname{NSCLC}(12,13)$.

PD-L1 has been reported to be expressed on meningioma $(24,25)$. In our studies (Figure 1C), meningiomas were stained from 1 patient each with grades I and II and 2 patients with grade III tumors, and PD-L1 expression appeared to increase with tumor grade. This confirms and extends the observations of Johnson et al. (25), who reported that overall PD-L1 expression in 58 FFPE meningiomas was 7\% and generally increased by grade: grade I, 3\% expressing PD-L1; grade II, 6\% expressing; and grade III, 18\% expressing. In addition, Du et al. (24) reported markedly greater levels of PD-L1 associated with meningioma: grade I, $40 \%$ expressing PD-L1; grade II, 60\% expressing; and grade III, 70\% expressing. Although Johnson et al. (25) suggested that these differences in overall expression could be due to the anti-PD-L1 antibody used, Gaule et al. (44) directly compared the clone used by Johnson (IHC22C3) and found it to yield concordant results with the antibody used in our studies (SP142). Importantly, although PD-L1 expression in other tumor types has been correlated with monotherapy antibody responses, it is not known what expression levels of PD-L1 are sufficient to be targeted by an ADCC-enabling antibody.

In several tumor types, PD-L1 transcription is increased during biologically relevant conditions of hypoxia (via HIF-1) or interferon response (via IRF1) $(33,45)$. Despite high basal levels, we found that meningioma tumor cells further upregulate PD-L1 under these conditions, and this enhanced tumor killing by the combination avelumab and haNK. Thus, targeting PD-L1 for ADCC may be particularly suited for conditions found in tumors from patients with malignant meningioma.

The degree of PD-L1 expression on target cells and the antibody binding affinity of cytotoxic effector cells contribute independently to ADCC efficacy (14). The haNK cell line provides an off-the-shelf source of NK cells that express the high-affinity CD16 receptor to mediate ADCC. This may be particularly germane for heavily treated patients with malignant meningioma who do not possess high numbers of NK cells.

Activated NK cells release immune inflammatory factors, including IFN- $\gamma$, that may tip the balance toward antitumor immunity by activating host immunity. High-grade meningiomas have an increased mutation burden compared with low-grade meningioma and are predicted to have a significantly larger 
neoantigen load that may increase immunoreactivity (46). A high neoantigen load combined with strong immune infiltration (24) supports the application of an immunotherapy-based therapeutic approach. Further, because meningioma treatment options include radiation, which may further increase neoantigen presentation, and minimal exposure to immunosuppressive chemotherapy, patients may have a sufficient immune repertoire to mount a robust antitumor response.

Here, we demonstrate that avelumab can target meningioma tumors for ADCC using avelumab and healthy donor NK cells. ADCC was further enhanced by haNK cells, which have recently demonstrated safety in humans. These considerations, in addition to location of meningiomas outside the bloodbrain barrier, support the design of a clinical trial using avelumab to target PD-L1-expressing meningioma tumors for ADCC by endogenous NK or haNK cells. This would potentially offer a novel therapeutic option for this challenging cancer with few treatment options and no proven effective chemotherapy.

\section{Methods}

Study design. Murine xenograft studies were designed to evaluate the effect of treatment among experimental groups. Power analyses were performed a priori with a 2-tailed, 2-sample $t$ test. Power was set to 0.8 and $\alpha$ of 0.05 to detect differences between group means of twice the observed SD. Mice were randomized based on tumor luminescence before treatment. Primary endpoints were survival and tumor-generated luminescence as a correlate for tumor size.

Cell culture and reagents. Meningioma cell lines IOMM-Lee, SAM, JEN, GAR, and CH-157 were generated in-house (University of Utah). Chordoma lines JHC7, UM-Chor1, U-CH1, and U-CH2 were obtained from the Chordoma Foundation (Durham, North Carolina, USA). Prostate cancer lines (LnCAP and PC3), breast cancer lines (MDA-MB-231 and MCF-7), and lung carcinoma lines (H460 and HCC4006) were obtained from ATCC. Growth medium was DMEM supplemented with $10 \%$ FBS and 1\% penicillin/ streptomycin. Healthy donor PBMCs were obtained from the NIH Clinical Center Blood Bank (protocol 99-CC-0618) and cryopreserved until use. NK cells were purified from cryopreserved healthy donor PBMCs using a negative selection NK cell isolation kit (STEMCELL Technologies or Miltenyi Biotec). Samples were genotyped for CD16 polymorphisms as described previously (14). haNK cells were provided by NantKwest (Culver City, California, USA) through a Cooperative Research and Development Agreement (CRADA) with the National Cancer Institute (NCI), NIH. haNK cells were cultured in phenol-free X-Vivo-10 medium (Lonza) with 5\% heat-inactivated human AB serum (Omega Scientific). Cells were incubated under $95 \%$ relative humidity at $5 \% \mathrm{CO}_{2}$. For hypoxia experiments, cells were cultured in Heracell VIOS $160 \mathrm{i} \mathrm{CO}_{2}$ incubators (Thermo Fisher Scientific) with $5 \% \mathrm{CO}_{2}$ and either $5 \%$ or $1 \%$ oxygen. For IFN- $\gamma$ treatment, cells were treated with $10 \mathrm{ng} / \mathrm{mL}$ recombinant human IFN- $\gamma$ (Peprotech) for 48 hours. Avelumab and the appropriate $\operatorname{IgG}_{1}$ isotype control were obtained from EMD Serono (a business of Merck Healthcare $\mathrm{KGaA}$ ) as part of a CRADA with the NCI. CD16 was blocked by incubating effector cells with anti-CD16 (clone B73.1, eBioscience) before ADCC assays.

CRISPR knockout. The sgRNA was designed according to the DNA sequence of CD274 exon 3 through http://crispr.mit.edu. Targeting sequence TGAACTGACATGTC was subcloned into the PX459 plasmid (Addgene 62988) and transfected into IOMM meningioma cells using Lipofectamine 3000 (Thermo Fisher Scientific). The following primer sequences were used: sgCD274.F (CACCGTGAACATGAACTGACATGTC), sgCD274.R (AAACGACATGTCAGTTCATGTTCAC), Surveyor. CD274.F (GGGGTACAAGAGCAGTGTTGT), and Surveyor.CD274.R (TGGTTTGGATGAATGGAGGTGA). IOMM-CD274-/- cells were sorted by flow cytometry and identified by the absence of PD-L1 surface staining. The knockout efficiency was confirmed using Surveyor assay, Sanger sequencing, Western blotting, and flow cytometry.

Antibody-dependent cellular cytotoxicity assay. ADCC assays were performed as previously described (14). In brief, trypsinized target cells were pulsed with ${ }^{111} \mathrm{In}$ for 20 minutes at $37^{\circ} \mathrm{C}$ and then washed to remove unbound ${ }^{111} \mathrm{In}$. Targets were incubated with avelumab or isotype antibody $(2 \mu \mathrm{g} / \mathrm{mL})$ (human $\operatorname{IgG}_{1}$, BE0297, BioXcell) and allowed to adhere in a 96-well, round-bottom plate (Corning Costar plate 3799 ) at 2000 targets/well. Healthy donor NK or haNK effector cells were added at the indicated E/T ratio, the plates were briefly centrifuged at $352 \mathrm{~g}$, and the ADCC assay was run for 4 hours at $37^{\circ} \mathrm{C}$. Supernatants were harvested and ${ }^{111}$ In was quantified using a WIZARD2 Automatic Gamma Counter (PerkinElmer). Spontaneous ${ }^{111}$ In release was quantified from supernatants of target cells incubated without effectors. Maximum ${ }^{111}$ In release was quantified from supernatants of target cells incubated with $0.05 \%$ 
Triton X-100. ADCC lysis was calculated as: percentage lysis $=([$ experimental $\mathrm{cpm}-$ spontaneous $\mathrm{cpm}) /$ (complete $\mathrm{cpm}-$ spontaneous cpm]) $\times 100$. ADCC assays were performed in RPMI 1640 medium supplemented with $10 \%$ FBS, $1 \%$ penicillin-streptomycin-glutamine, $1 \%$ sodium pyruvate, $1 \%$ nonessential amino acids, and $15 \mathrm{mM}$ HEPES.

Flow cytometry. Single-cell suspensions were washed, resuspended in PBS/BSA 1\%, and counted using a Cellometer Auto 2000 cell counter (Nexcelom). Viability was determined with trypan blue counterstaining or fixable viability dye eFluor780 (eBioscience) in PBS. Single-cell suspensions were washed, resuspended in PBS/BSA 1\%, and counted using a Cellometer Auto 2000 cell counter (Nexcelom). Viability was determined with trypan blue counterstaining.

Before antibody staining, cell suspensions were incubated with human Fc block (catalog 564219, BD Biosciences) for 10 minutes. Surface staining was performed in PBS/BSA 1\% for 30 minutes on ice with CD274-APC or CD274-PE (clone 29E.2A3, catalog 329708,BioLegend), CD279-APC (clone EH12.2H7, catalog 329908, BioLegend), CD80-APC (clone 2D10, catalog 305220, BioLegend), CD158-APC (clone HP-MA4, catalog 339510, BioLegend), CD94-APC (clone DX22, catalog 305508, BioLegend), CD66a/c/e (clone ASL-32, catalog 342308, BioLegend), MIC A/B-APC (clone 6D4, catalog 320908, BioLegend), and HLA-ABC-APC (clone W6/32, catalog 311410, BioLegend). Corresponding isotypes were used for negative controls. Cells were fixed with Cytofix Fixation Buffer (catalog 554655, BD Biosciences) for 15 minutes, followed by washing with PBS/BSA 1\% before sample acquisition. Samples were acquired on a FACSCalibur flow cytometer (BD Biosciences) and analyzed with FlowJo version 10.5.3 (TreeStar Inc.).

Western blots. Tumor cells were lysed using RIPA buffer with a protease inhibitor cocktail (MilliporeSigma). Lysates were separated by SDS-PAGE (Bio-Rad) and transferred to PVDF membranes. Membranes were probed for PD-L1 (E1L3N) and $\beta$-actin (8H10D10) from Cell Signaling Technology.

Mice and tumor lines. Female athymic nude mice, 6-8 weeks old, were purchased from Charles River and maintained in pathogen-free microinsulator cages. For orthotopic meningioma models, $1 \times 10^{4}$ IOMM meningioma cells were injected as single-cell suspensions at the skull base. Avelumab (200 $\mu \mathrm{g} /$ mouse $)$ and haNK cells $\left(1 \times 10^{7}\right.$ cells/mouse $)$ were injected intravenously on Mondays, Wednesdays, and Fridays for 2 weeks. Before in vitro or in vivo use, haNK cells were lethally irradiated (10 Gy) to block cellular proliferation. Importantly, irradiation did not impair IL-2 production or cytotoxic function $(22,47)$. For subcutaneous models, $5 \times 10^{6}$ IOMM meningioma cells were injected subcutaneously on the flank. Tumor lines were stably transduced with firefly-luciferase, permitting luminescence reading as a proxy for tumor size. Mice were injected with $3 \mathrm{mg}$ luciferin (GoldBio), and images were analyzed with Living Image software (Xenogen Corporation). Flank tumor models were measured with calipers, and tumor volume was calculated with an ellipsoid formula: (length $\times$ width $^{2} \times \pi$ ) $/ 6$. All studies were conducted under protocols approved by the NCI Animal Care and Use Committee.

Immunohistochemistry. Five-micrometer serial sections were prepared from FFPE xenograft tissues. Immunohistochemistry staining for GZMB (NB100-684, Novus Biologicals) and cleaved caspase-3 (Asp175, Cell Signaling Technology) were performed by HistoServ, Inc., using standard procedure. In brief, tissue sections were deparaffinized and rehydrated in xylene and ethanol, respectively. Sections were then subjected to heat-induced antigen retrieval at $95^{\circ} \mathrm{C}$ for 20 minutes and incubated with primary antibody overnight at $4^{\circ} \mathrm{C}$. HRP-conjugated anti-rabbit IgG or anti-mouse IgG secondary antibody were incubated with the specimens and developed by DAB substrate. Immunohistochemistry staining for PD-L1 (SP142, 1:100, Spring Bioscience) of FFPE meningioma tissue blocks were performed on the automated Leica Bond-Max (Leica Biosystems) according to the manufacturer's instructions. Sections were stained alongside a positive control of normal human placenta (HuFPT080, US Biomax, Inc.). Tissue sections underwent citrate antigen retrieval for 20 minutes at $98^{\circ} \mathrm{C}$ before antibody staining. Images were captured using Nikon Eclipse CIE light microscope. A standard H\&E staining was used for histopathological evaluation.

Statistics. Statistical analyses were performed using GraphPad Prism 7.0 software. Data are expressed as mean $\pm \mathrm{SD}$ or the SEM as indicated. Statistical significance was evaluated by $t$ test, ANOVA, or unequal-variance (Welch's) ANOVA. $P$ values less than 0.05 were considered significant.

Study approval. Animal studies were conducted under NIH IACUC approval NOB-007. Informed consent was not required for use of archival samples. 


\section{Author contributions}

AJG designed research studies, conducted experiments, acquired and analyzed data, and wrote the manuscript. SH conducted experiments, acquired and analyzed data, and contributed to writing the manuscript. MP, HS, WZ, JL, VS, YL, JJ, XC, and RF conducted experiments and acquired and analyzed data. RJ and DG provided reagents. JS provided reagents and edited the manuscript. MRG designed research studies and edited the manuscript. EKN provided reagents and edited the manuscript. CY conducted experiments, analyzed data, provided reagents, and edited the manuscript. JHL provided reagents, reviewed data, and reviewed the manuscript. PSS provided reagents, reviewed data, and reviewed the manuscript. JWH designed research studies, analyzed data, provided reagents, and edited the manuscript. DMP designed research studies and analyzed data.

\section{Acknowledgments}

We would like to thank Kenneth Aldape for guidance in meningioma histopathological evaluation and Debra Weingarten for her assistance in the preparation of this manuscript. This research was supported by the Intramural Research Program of the Center for Cancer Research, NCI, NIH.

Address correspondence to: James W. Hodge, National Cancer Institute, Building 10, Room 8B13, 9000 Rockville Pike, Bethesda, Maryland 20892, USA. Phone: 240.858.3466; Email: hodgej@mail.nih.gov.

1. Ostrom QT, et al. CBTRUS statistical report: primary brain and central nervous system tumors diagnosed in the United States in 2007-2011. Neuro-oncology. 2014;16(Suppl 4):iv1-i63.

2. Aghi MK, et al. Long-term recurrence rates of atypical meningiomas after gross total resection with or without postoperative adjuvant radiation. Neurosurgery. 2009;64(1):56-60.

3. Wang YC, et al. Long term surgical outcome and prognostic factors of atypical and malignant meningiomas. Sci Rep. 2016;6:35743

4. Yang SY, Park CK, Park SH, Kim DG, Chung YS, Jung HW. Atypical and anaplastic meningiomas: prognostic implications of clinicopathological features. J Neurol Neurosurg Psychiatry. 2008;79(5):574-580.

5. Cao X, et al. Survival rates, prognostic factors and treatment of anaplastic meningiomas. J Clin Neurosci. 2015;22(5):828-833.

6. Sughrue ME, Sanai N, Shangari G, Parsa AT, Berger MS, McDermott MW. Outcome and survival following primary and repeat surgery for World Health Organization Grade III meningiomas. J Neurosurg. 2010;113(2):202-209.

7. Hoos A. Development of immuno-oncology drugs - from CTLA4 to PD1 to the next generations. Nat Rev Drug Discov. 2016;15(4):235-247.

8. Powles T, et al. MPDL3280A (anti-PD-L1) treatment leads to clinical activity in metastatic bladder cancer. Nature. 2014;515(7528):558-562.

9. Balar AV, et al. Atezolizumab as first-line treatment in cisplatin-ineligible patients with locally advanced and metastatic urothelial carcinoma: a single-arm, multicentre, phase 2 trial. Lancet. 2017;389(10064):67-76.

10. Massard C, et al. Safety and efficacy of durvalumab (MEDI4736), an anti-programmed cell death ligand-1 immune checkpoint inhibitor, in patients with advanced urothelial bladder cancer. J Clin Oncol. 2016;34(26):3119-3125.

11. Brahmer JR, et al. Safety and activity of anti-PD-L1 antibody in patients with advanced cancer. N Engl J Med. 2012;366(26):2455-2465.

12. Heery CR, et al. Avelumab for metastatic or locally advanced previously treated solid tumours (JAVELIN Solid Tumor): a phase 1a, multicohort, dose-escalation trial. Lancet Oncol. 2017;18(5):587-598.

13. Gulley JL, et al. Avelumab for patients with previously treated metastatic or recurrent non-small-cell lung cancer (JAVELIN Solid Tumor): dose-expansion cohort of a multicentre, open-label, phase 1b trial. Lancet Oncol. 2017;18(5):599-610.

14. Fujii R, et al. Enhanced killing of chordoma cells by antibody-dependent cell-mediated cytotoxicity employing the novel antiPD-L1 antibody avelumab. Oncotarget. 2016;7(23):33498-33511.

15. Boyerinas B, et al. Antibody-dependent cellular cytotoxicity activity of a novel anti-PD-L1 antibody avelumab (MSB0010718C) on human tumor cells. Cancer Immunol Res. 2015;3(10):1148-1157.

16. Bibeau F, et al. Impact of Fc $\gamma$ RIIa-Fc $\gamma$ RIIIa polymorphisms and KRAS mutations on the clinical outcome of patients with metastatic colorectal cancer treated with cetuximab plus irinotecan. J Clin Oncol. 2009;27(7):1122-1129.

17. Cartron G, et al. Therapeutic activity of humanized anti-CD20 monoclonal antibody and polymorphism in IgG Fc receptor Fc $\gamma$ RIIIIa gene. Blood. 2002;99(3):754-758.

18. Mellor JD, Brown MP, Irving HR, Zalcberg JR, Dobrovic A. A critical review of the role of Fc gamma receptor polymorphisms in the response to monoclonal antibodies in cancer. J Hematol Oncol. 2013;6:1.

19. Musolino A, et al. Immunoglobulin G fragment C receptor polymorphisms and clinical efficacy of trastuzumab-based therapy in patients with HER-2/neu-positive metastatic breast cancer. J Clin Oncol. 2008;26(11):1789-1796.

20. Zhang W, et al. FCGR2A and FCGR3A polymorphisms associated with clinical outcome of epidermal growth factor receptor expressing metastatic colorectal cancer patients treated with single-agent cetuximab. J Clin Oncol. 2007;25(24):3712-3718.

21. Boissel L, et al. Retargeting NK-92 cells by means of CD19- and CD20-specific chimeric antigen receptors compares favorably with antibody-dependent cellular cytotoxicity. Oncoimmunology. 2013;2(10):e26527.

22. Gong JH, Maki G, Klingemann HG. Characterization of a human cell line (NK-92) with phenotypical and functional characteristics of activated natural killer cells. Leukemia. 1994;8(4):652-658.

23. Yan Y, et al. Antileukemia activity of a natural killer cell line against human leukemias. Clin Cancer Res. 1998;4(11):2859-2868. 
24. Du Z, et al. Increased expression of the immune modulatory molecule PD-L1 (CD274) in anaplastic meningioma. Oncotarget. 2015;6(7):4704-4716

25. Johnson MD. PD-L1 expression in meningiomas. J Clin Neurosci. 2018;57:149-151.

26. Benson DM, et al. The PD-1/PD-L1 axis modulates the natural killer cell versus multiple myeloma effect: a therapeutic target for CT-011, a novel monoclonal anti-PD-1 antibody. Blood. 2010;116(13):2286-2294.

27. Dong W, et al. The mechanism of anti-PD-L1 antibody efficacy against PD-L1-negative tumors identifies NK cells expressing PD-L1 as a cytolytic effector. Cancer Discov. 2019;9(10):1422-1437.

28. Hsu J, et al. Contribution of NK cells to immunotherapy mediated by PD-1/PD-L1 blockade. J Clin Invest. 2018;128(10):4654-4668.

29. Liu Y, et al. Increased expression of programmed cell death protein 1 on NK cells inhibits NK-cell-mediated anti-tumor function and indicates poor prognosis in digestive cancers. Oncogene. 2017;36(44):6143-6153.

30. Nayyar G, Chu Y, Cairo MS. Overcoming resistance to natural killer cell based immunotherapies for solid tumors. Front Oncol. 2019;9:51.

31. Koene HR, Kleijer M, Algra J, Roos D, von dem Borne AE, de Haas M. Fc gammaRIIIa-158V/F polymorphism influences the binding of IgG by natural killer cell Fc gammaRIIIa, independently of the Fc gammaRIIIa-48L/R/H phenotype. Blood. 1997;90(3):1109-1114.

32. Ruf M, Moch H, Schraml P. PD-L1 expression is regulated by hypoxia inducible factor in clear cell renal cell carcinoma. Int $J$ Cancer. 2016;139(2):396-403.

33. Noman MZ, et al. PD-L1 is a novel direct target of HIF-1 $\alpha$, and its blockade under hypoxia enhanced MDSC-mediated T cell activation. J Exp Med. 2014;211(5):781-790.

34. Reszec J, Rutkowski R, Chyczewski L. The expression of hypoxia-inducible factor-1 in primary brain tumors. Int J Neurosci. 2013;123(9):657-662.

35. Bhowmik A, Khan R, Ghosh MK. Blood brain barrier: a challenge for effectual therapy of brain tumors. Biomed Res Int. 2015;2015:320941.

36. Thompson $\mathrm{RH}$, et al. Tumor B7-H1 is associated with poor prognosis in renal cell carcinoma patients with long-term follow-up. Cancer Res. 2006;66(7):3381-3385.

37. Hamanishi J, et al. Programmed cell death 1 ligand 1 and tumor-infiltrating CD8+ T lymphocytes are prognostic factors of human ovarian cancer. Proc Natl Acad Sci U S A. 2007;104(9):3360-3365.

38. Jesinghaus M, et al. Increased intraepithelial CD3+ T-lymphocytes and high PD-L1 expression on tumor cells are associated with a favorable prognosis in esophageal squamous cell carcinoma and allow prognostic immunogenic subgrouping. Oncotarget. 2017;8(29):46756-46768.

39. Xue S, Song G, Yu J. The prognostic significance of PD-L1 expression in patients with glioma: A meta-analysis. Sci Rep. 2017;7(1):4231.

40. Rittmeyer A, et al. Atezolizumab versus docetaxel in patients with previously treated non-small-cell lung cancer (OAK): a phase 3, open-label, multicentre randomised controlled trial. Lancet. 2017;389(10066):255-265.

41. Inman BA, Longo TA, Ramalingam S, Harrison MR. Atezolizumab: a PD-L1-blocking antibody for bladder cancer. Clin Cancer Res. 2017;23(8):1886-1890.

42. Wurz GT, Kao CJ, DeGregorio MW. Novel cancer antigens for personalized immunotherapies: latest evidence and clinical potential. Ther Adv Med Oncol. 2016;8(1):4-31.

43. Donahue RN, et al. Analyses of the peripheral immunome following multiple administrations of avelumab, a human IgG1 antiPD-L1 monoclonal antibody. J Immunother Cancer. 2017;5:20.

44. Gaule P, et al. A quantitative comparison of antibodies to programmed cell death 1 ligand 1. JAMA Oncol. 2017;3(2):256-259.

45. Garcia-Diaz A, et al. Interferon receptor signaling pathways regulating PD-L1 and PD-L2 expression. Cell Rep. 2017;19(6):1189-1201.

46. Bi WL, et al. Genomic landscape of high-grade meningiomas. NPJ Genom Med. 2017;2:15.

47. Jochems C, et al. ADCC employing an NK cell line (haNK) expressing the high affinity CD16 allele with avelumab, an antiPD-L1 antibody. Int J Cancer. 2017;141(3):583-593. 